\title{
Real-Time EMG driven Lower Limb Actuated Orthosis for Assistance As Needed Movement Strategy
}

\author{
Walid Hassani, Samer Mohammed, Yacine Amirat \\ LISSI Lab, University Paris Est Créteil (UPEC) \\ Vitry Sur Seine, 94400, France \\ Email: \{walid.hassani,samer.mohammed,amirat\}@u-pec.fr
}

\begin{abstract}
This paper presents a new approach to control a wearable knee joint exoskeleton driven through the wearer's intention. A realistic bio-inspired musculoskeletal knee joint model is used to control the exoskeleton. This model takes into account changes in muscle length and joint moment arms as well as the dynamics of muscle activation and muscle contraction during lower limb movements. Identification of the model parameters is done through an unconstrained optimization problem formulation. A control law strategy based on the principle of assistance as needed is proposed. This approach guarantees asymptotic stability of the knee joint orthosis and adaptation to human-orthosis interaction. Moreover, the proposed control law is robust with respect to external disturbances. Experimental validations are conducted online on a healthy subject during flexion and extenion of their knee joint. The proposed control strategy has shown satisfactory performances in terms of tracking trajectory and adaptation to human tasks completion.
\end{abstract}

\section{INTRODUCTION}

Exoskeletons are mainly used in assisting and rehabilitating dependent people as well as in human power augmentation. They have known a huge expansion in the last decade as they are continually attracting the scientific and the medical community due to the continuous increasing rate of dependent people [16], [17]. Research in this field has started in the sixties and has tended to assist depending people by coupling the exoskeleton with their limbs in the aim of allowing the wearer to regain natural movements [8]. The key issue when dealing with wearable exoskeletons concerns the interaction between wearer and the exoskeleton from a physical or a cognitive point of view and ideally from both [19]. The most developed exoskeleton research projets are based on physical interaction, through force interfaces, whether by imposing predefined movements [28, 13], or by using forces sensors between the exoskeleton and the wearer and/or the inverse dynamics of the wearer body $[8,20,5,6]$. One of the major drawbacks of this physical interaction mode is the poorness reactivity of the exoskeleton caused by the time delay between the wearer's intention estimation and the control input applied to the exoskeleton [4]. This can be very tiring particularly for dependent people. Moreover, this interaction mode requires the use of an accurate inverse dynamics model, that is very sensitive to the human anthropomorphic parameters.

An alternative approach is the cognitive human-exoskeleton interaction that allows a closed loop interaction schema ac- cording to the three following steps: the first one concerns the reasoning and planning tasks (brain activities), the second one is related to muscle activity measurements through the Electromyography (EMG) and the third one concerns the generation of the assisting exoskeleton movements [19]. The cognitive interaction mode is biologically inspired and is done through neural excitation of given skeletal muscles. This approach takes full advantage of the human natural control mechanisms [19]. However, such interaction mode is a complex mechanism because it requires; (i) an efficient processing of the EMG signals that are in general noisy, (ii) an accurate estimation of the wearer's intention, i.e, the necessary torque to achieve a given desired movement. This estimation needs a precise and realistic musculoskeletal modeling of the assisted joint, (iii) a balancing strategy between the wearer's contribution and the exoskeleton's one such that the exoskeleton will be able to compensate the deficiency in the human generated torque. To the authors' best knowledge, only few works have addressed together the issues listed above. EMG-driven approaches can be classified as: physical-based approaches [24, 4] and non-physical-based approaches [26, 10, 9, 7, 15]. The physically-based approaches are more realistic as they use musculoskeletal model to estimate muscles forces and joint torques. In [24, 23], a fixed upper limb exoskeleton is used to assist the flexion/extention movements of the elbow joint. The wearer's force is evaluated using EMG and a simplified Hill-type muscle model is used along with the use of neural networks. The assisting torque provided by the exoskeleton is linearly dependent of the wearer's torque. However, the parametric identification as well as the experimental validation of the musculoskeletal model were not provided. In [4], a knee joint exoskeleton controlled through the wearer's intention estimation is proposed. A musculoskeletal knee joint model is introduced to improve the estimation of the wearer's torque. Two control schemes were used; in the first one, the estimated wearer's torque is linearly dependent with respect to the assisting torque, while in the second one, the wearer's movement is estimated using the knee joint torque of the wearer and its inverse rigid-body dynamics. In [26], the authors proposed a one DOF wearable powered ankle foot orthosis with artificial pneumatic muscle for gait rehabilitation. The control schema is based on the assisting torque estimation by using a propor- 
tional controller as a function of the EMG activation level. An EMG interface to control two degrees of freedom exoskeleton is presented in [10]. This exoskeleton assists flexion/extention and pronation/supination shoulder joint to perform predefined movements. The controller output is only moderated using raw EMG patterns previously learned using fuzzy-neuro model. Authors in [9] used raw EMG pattern recognition to control a two DOF wrist exoskeleton. A support vector machine is used to classify the EMG signals and to estimate the wearer's intention. The estimated torque is shared linearly to assist the wearer's movements. The Hybrid Assistive Limb (HAL) relies on the detection of motion intention and the achievement of the movement task. The so-called voluntary control system estimates the wearer's intention through the detection of the nerve signals (EMG) [15] 7].

In the litterature, different works have dealt with the control of exoskeletons. In [8], the authors proposed a control approach based on a scaled compensation of the estimated exoskeleton dynamics. In [30, 32], a PD controller is used to stabilize the body of the wearer by guaranteeing balance of the gravity effect. A PD controller is also developed in [2] to ensure gravity, coriolis and friction forces compensation. A fictitious gain coupled with a proportional-integrator-derivative controller (PID) is introduced in [11]. This controller is able to take into account the wearer's body dynamics during walking, load carrying, etc.

Most of the aforecited works rely on the use of proportional derivative based controllers that are widely used to control exoskeletons, but very few studies have dealt with the exoskeleton's stability.

The contributions of this study can be listed as follows: Firstly, a realistic bio-inspired musculoskeletal model [3] is used to control the orthosis movements. Parameters such as muscle length variations, activation and contraction dynamics and moment arms are taken into account. This will allow a better estimation of the wearer's intention. The identification of the above parameters was made through an unconstrained optimization problem formulation. Secondly, a control law strategy is developed to guarantee asymptotic stability of the knee joint orthosis. The exoskeleton is controlled upon the wearer's intention using a control strategy that has shown robustness with respect to external disturbances. Finally, experimental validations of the proposed strategy are conducted with a young healthy subject upon an experimental protocol. The rest of this paper is organized as follows: section II presents the musculoskeletal modeling and the control law strategy. Section III shows the parametric identification procedure. Experimental results of the proposed control strategy are given in section IV. Conclusion and future works are shown in section V.

\section{EXOSKELETON-WEARER MODELING AND CONTROL}

\section{A. Wearer-Exoskeleton Modeling}

The wearer is in a sitting position performing flexion and extension movements of the knee joint without taking into account the ground contact. The exoskeleton is supposed to be rigidly attached to the wearer's leg such that the whole system rotates synchronously about the knee joint. The shank foot orthosis is controlled by both the human torque resulting from muscles contraction and the orthosis generated torque [22], [21]. The motion equation is given by:

$$
J \ddot{\theta}+B \dot{\theta}+\frac{\partial E_{p}}{\partial \theta}=\tau_{e}+\tau_{h}
$$

Where:

$$
\begin{gathered}
\tau_{h}=\sum_{j=1}^{8} F_{j}^{m t}\left(E M G_{j}, \theta\right) r_{j}^{m t} \\
E_{p}=K \frac{1}{2}\left(\theta-\theta_{r}\right)^{2}-\tau_{g} \cos \left(\theta-\theta_{r}\right)
\end{gathered}
$$

$J$ and $B$ represent respectively the inertia and the viscous damping coefficient of the shank-foot-exoskeleton, $\tau_{h}$ represents the wearer's developed torque, $F_{j}^{m t}$ and $r_{j}^{m t}$ are the force and the moment arm of the $j^{\text {th }}$ muscle tendon. $J$ is supposed to be constant during flexion/extension of the knee joint. The following muscles are considered (Fig 1) to estimate the knee joint torques: rectus femoris (RF), vastus lateralis (VL), vastus medialis (VM) and vastus intermedius (VI) as quadriceps for knee joint extension and semi-tendinous (ST), semi-membranous (SM), biceps femoris long head (BL)/short head (BS) as hamstrings for knee joint flexion, $\tau_{e}$ shows the exoskeleton generated torque; $\theta, \dot{\theta}$ and $\ddot{\theta}$ represent respectively the exoskeleton-wearer position, velocity and acceleration; $E_{p}$ shows the potential energy of the system; $K$ gives the knee joint stiffness; $\theta_{r}$ represents the knee joint angle at rest position and $\tau_{g}$ shows the gravitational torque.

The muscle models used in this study are based on the modified Stiff tendon Hill type model [25]. This model consists of a force-length $\left(f_{l}\right)$ and force-velocity $\left(f_{v}\right)$ relationships [31] as well as a parallel viscous damping element $\left(f_{d}\right)$ [27]. Both relationships are normalized respectively to the maximum isometric muscle force $\left(F_{\max }\right)$, the muscle optimal fiber length $l_{o}^{m}$ and the maximum muscle contraction velocity $\left(v_{\max }\right)$ [35]. The force produced by the muscle tendon model $F^{m t}(t)$ is expressed by the following equation:

$$
\begin{gathered}
F^{m t}(t)=F_{\text {max }}\left(a(t) f_{l}\left(\bar{l}^{m}(t)\right) f_{v}\left(\bar{v}^{m}(t)\right)+f_{d}\left(\bar{v}^{m}(t)\right)\right) \\
\dot{a}(t)+\frac{1}{\tau_{a c t}}\left[\frac{\tau_{a c t}}{\tau_{\text {deact }}}+\left(1-\frac{\tau_{a c t}}{\tau_{\text {deact }}}\right) c(t)\right] a(t)=\frac{1}{\tau_{a c t}} c(t) \\
f_{l}\left(\bar{l}^{m}(t)\right)=\exp \left(-\left(\bar{l}^{m}(t)-1\right)^{2} / \gamma\right) \\
f_{v}\left(\bar{v}^{m}\right)=\left\{\begin{array}{l}
\frac{0.3\left(\bar{v}^{m}(t)+1\right)}{-\bar{v}^{m}(t)+0.3} \\
\frac{\left(2.34 \bar{v}^{m}(t)+0.039\right)}{1.3 \bar{v}^{m}(t)+0.039} \quad \bar{v}^{m}(t) \leq 0 \\
f_{d}\left(\bar{v}^{m}(t)\right)=B^{m} \bar{v}^{m}(t)
\end{array}\right.
\end{gathered}
$$




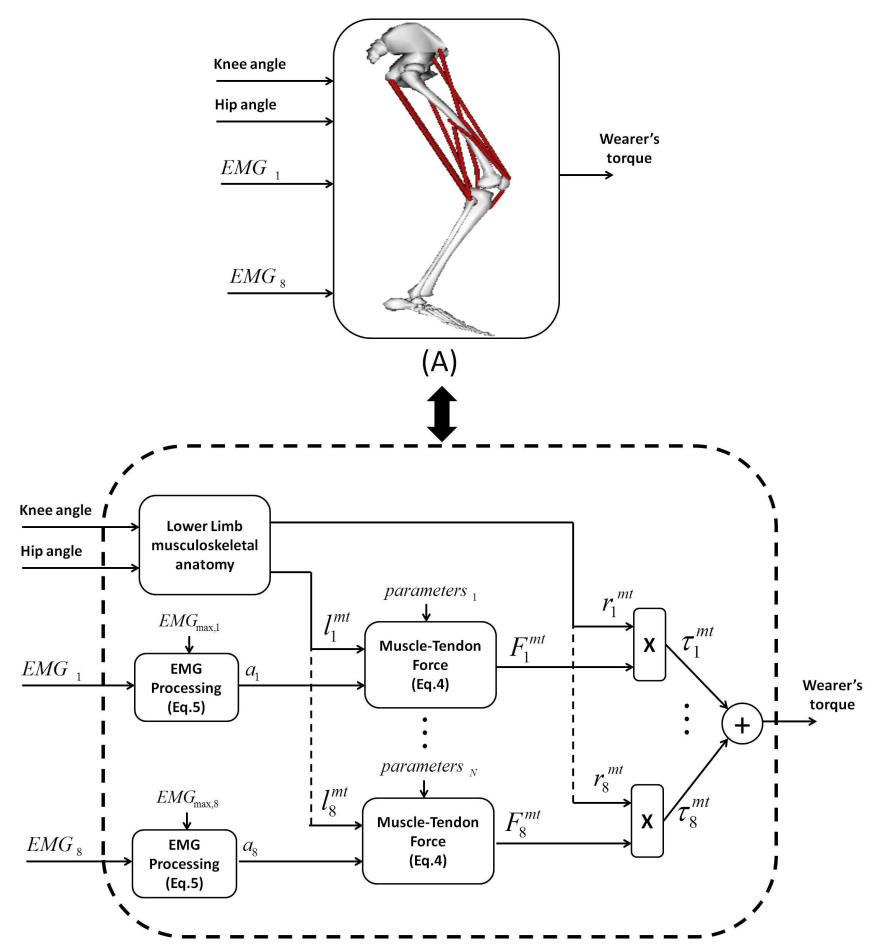

(B)

Fig. 1. Wearer's Torque Estimation

Where $a(t)$ represents the muscle activation [34]; $c(t)$ is the resulting signal from filtering, full-wave rectification and normalization to $E M G_{\max }$ that is the maximal recorded value of EMG during the muscle's maximal voluntary contraction; $\tau_{\text {act }}$ and $\tau_{\text {deact }}$ represent the muscle's activation time constant and the deactivation time constant respectively; $B^{m}$ represents the muscle damping; $\bar{l}^{m}(t)$ and $\bar{v}^{m}(t)$ show the instantaneous normalized muscle fiber length and normalized muscle fiber velocity with respect to $l_{o}^{m}$ and $v_{\max }$ respectively; $t$ represents the time variable. $\gamma$ in (6) represents a shape factor and is equal to 0.45 [31]. Numerical values used in (7) represent the features of the force-velocity relationship such as curvature, maximum normalized force, etc. [27]. The tendon is assumed sufficiently stiff and the muscle fiber length $l^{m}(t)$ is expressed as follows:

$$
l^{m}(t)=\left(l^{m t}(t)-l_{s}^{t}\right)
$$

$l_{s}^{t}$ is the tendon slack length and $l^{m t}(t)$ is the instantaneous muscle tendon length computed using the lower limb musculoskeletal model based on the use of the anatomical data reported in [3, 1]; this model includes the tibia, the femur, the patella and the pelvis body segments. This anatomical model consists of line segments models of the 8 above-mentioned muscle tendon units spanning the knee joint.

\section{B. Exoskeleton Control}

This section describes the interaction between the exoskeleton and the wearer. An "Assistance as Needed" approach is

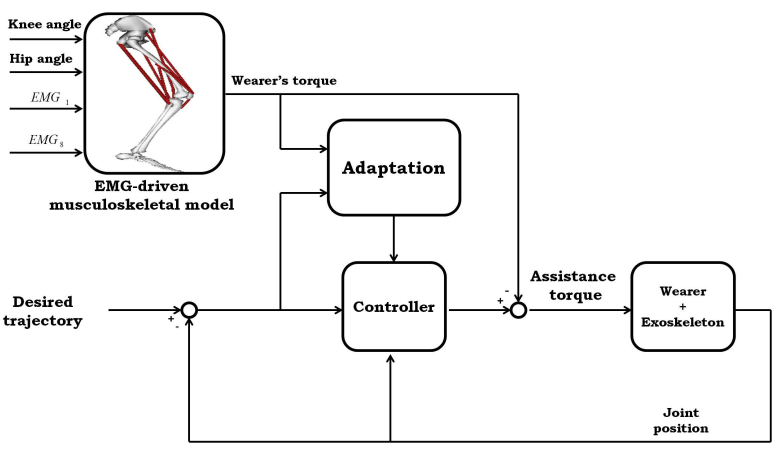

Fig. 2. General Control Layout

developed (Fig. 2) to allow a flexible and smooth exoskeleton/wearer interaction. This approach takes into account the subject's intention through the human developed torque in order to control the exoskeleton under a stable passivity based control strategy [29]. The controller's parameters are adapted automatically with respect to the wearer's changing human contribution during the completion of a given task. This approach can be used particularly during a rehabilitation process as the contribution of the exoskeleton torque decreases with the improvement in the performance of the wearer. Indeed, the controller continuously attempt to reduce its assistance ratio when the wearer develops an effort to accomplish a given task, on the other side, when the user moves largely away of the desired joint trajectory, the controller develops a countertorque to bring the wearer limb back to the desired trajectory or to its vicinity.

In this study, the proposed control law strategy is based on the use of the passivity-based approach used particularly for nonlinear time-varying systems [18, 29]. The main advantage of this approach is to reduce the order of the closed loop system by introducing a virtual error $s=\dot{e}+\lambda e$ that is driven to zero in finite time.

The control torque and stability analysis are presented in the following:

Proposition: Consider the knee-joint human-exoskeleton model described by (1) with $\theta$ and $\dot{\theta}$ representing respectively the knee joint angle and angular velocity. $\theta_{d}$ denotes the desired knee joint position and $e=\theta-\theta_{d}$ the tracking error. $\dot{\theta}_{d}$ shows the desired velocity and $\dot{e}=\dot{\theta}-\dot{\theta}_{d}$ the tracking velocity error. The control torque is defined by:

$$
\tau_{e}=J\left(\ddot{\theta}_{d}-(\dot{\lambda e})\right)+B\left(\dot{\theta_{d}}-\lambda e\right)+\frac{\partial E_{p}}{\partial \theta}-K_{d} s-\tau_{h}
$$

with: $s=\dot{e}+\lambda e$

Then, $\dot{s}=\ddot{e}+(\dot{\lambda e})=\ddot{e}+\dot{\lambda} e+\lambda \dot{e}$

$$
\lambda=\lambda_{0} \frac{1+K_{1}\|e\|}{1+K_{2}\left\|\tau_{h}-\tau_{h, d}\right\|}
$$

Where $s$ denotes a virtual error, $\tau_{h, d}=\sum_{1}^{8} F_{\max , j} f_{d, j} r_{j}^{m t}$ the wearer's passive damping torque, $\lambda_{0}, K_{d}, K_{1}$ and $K_{2}$ are positive scalar parameters. The passive torque $\tau_{h, d}$ is 
subtracted from $\tau_{h}$ in order to take into account only the active wearer's developed efforts. During the rehabilitation task, the wearer is asked to develop efforts [14], in order to move his/her limbs along a desired trajectory. Hence, the torque provided by the exoskeleton is adapted upon the wearer performance such that if the wearer deviates from the desired trajectory, the exoskeleton should generate a restoring force. The exoskeleton's developed torque is proportional to the virtual error $s$. From (11), one can notice that if the wearer approaches the desired trajectory, $e$ decreases, $\tau_{h}$ increases and consequently $\lambda$ decreases. On the other hand, if the wearer deviates from the desired trajectory $e$ and $\tau_{h}$ increase. In order to increase $\lambda$, appropriate values of $K_{1}$ and $K_{2}$ should be used. Proof: Let's define the following Lyapunov function $\mathrm{V}$ positive definite and radially unbounded:

$$
V=\frac{1}{2} J s^{2}
$$

Using (11) and (10), the closed loop system can be expressed as follows:

$$
\begin{gathered}
J(\ddot{e}+\lambda \dot{e}+\dot{\lambda} e)=-B(\dot{e}+\lambda e)-K_{d} s \\
J \dot{s}=-B s-K_{d} s=-\left(B+K_{d}\right) s
\end{gathered}
$$

The time derivative of (12) is:

$$
\dot{V}=J \dot{s} s
$$

Combining (15) and (14), $\dot{V}$ can be expressed as follows:

$$
\dot{V}=-\left(B+K_{d}\right) s^{2} \leq 0
$$

The Lyapunov function $V$ decreases till the variable $s$ reaches the origin $s=0$, then:

$$
\dot{e}=-\lambda e=-\lambda_{0} \frac{1+K_{1}\|e\|}{1+K_{2}\left\|\tau_{h}-\tau_{h, d}\right\|} e
$$

To prove the decrease of the error $e$, consider the following energy function:

$$
L=\frac{1}{2} e^{2}
$$

The time derivative of the $L$ is given as follows:

$$
\dot{L}=e \dot{e}=-\lambda e^{2}=-\lambda_{0} \frac{1+K_{1}\|e\|}{1+K_{2}\left\|\tau_{h}-\tau_{h, d}\right\|} e^{2} \leq 0
$$

Since $\lambda_{0}, K_{1}$ and $K_{2}$ are greater than 0 , then, the energy function $L$ decreases until it reaches 0 . Therefore, the error $e$ tends to zero and $(\theta, \dot{\theta})=\left(\theta_{d}, \dot{\theta}_{d}\right)$ is an asymptotically stable point of the closed-loop system.

\section{PARAMETRIC IDENTIFICATION}

\section{A. Identification procedure}

The identification process is done through two steps; the first one concerns the identification of the inertial parameters of the shank-foot orthosis and the second steps concerns the identification of the muscle-tendon parameters. a) Step I: The parameters $J, B, K$ and $\tau_{g}$ vary from a wearer to another. They have to be identified for each one. $\tau_{g}$ depends on the wearer shank-foot weight and can be estimated according to the regression equations proposed by Winter [33]. $K$ is identified in static conditions, the shank-foot orthosis moves by a step of $15^{\circ}$ from $-80^{\circ}$ to $0^{\circ}$. Velocity $\dot{\theta}$ and acceleration $\ddot{\theta}$ of the shank foot orthosis are supposed to be equal to zero at static conditions. Thus, equation (1) can be expressed as follows:

$$
K\left(\theta-\theta_{r}\right)=\tau_{e}-\tau_{g} \sin \left(\theta-\theta_{r}\right)
$$

$J$ and $B$ are identified offline in dynamic conditions using linear least square optimization method. The wearer's legfoot is moved by the exoskeleton to perform a given desired movement with a frequency range that covers the daily activities living leg movements. Particularly, the frequencies of the desired movements used in this study range between $0.01 \mathrm{~Hz}$ and $0.3 \mathrm{~Hz}$. The wearer is asked to not develop any muscular contractions during this step. Equation (1) in a dynamic context can be formulated as follows :

$$
J \ddot{\theta}+B \dot{\theta}=\tau_{e}-\tau_{g} \sin \left(\theta-\theta_{r}\right)-K\left(\theta-\theta_{r}\right)
$$

b) Step II: This step concerns the identification of the muscle-tendon parameters, particularly, $l_{o}^{m}, l_{s}^{t}, F_{\max }$ parameters that correspond to the set of muscles involved in the knee joint flexion and extension. The muscle-tendon length $l^{m t}$ as well as the muscle-tendon moment arm $r^{m t}$ have to be scaled, since it is assumed that $r^{m t}$ is equal to $\frac{\partial l^{m t}}{\partial \theta}$ [3]. Both $l^{m t}$ and $r^{m t}$ are position $(\theta)$ dependent. A variable $S_{c}$ is used to scale both $l^{m t}$ and $r^{m t}$. The muscle force $F^{m t}$ is estimated using (44. Muscle-tendon parameters are identified for both flexor and extensor muscles separately according to the following optimization formulation:

$$
\underset{\chi}{\operatorname{minimize}} \frac{1}{N} \sum_{i=1}^{N}\left(\tau(i)-\sum_{j=1}^{4} F_{j}^{m t}(i) r_{j}^{m t}(i)\right)^{2}
$$

Where $\chi=\left[\chi_{1}, \chi_{3}, \chi_{3}, \chi_{4}\right]$ is the parameter's vector to be identified, $\tau$ represents the wearer's generated torque estimated using (1), with:

$$
\chi_{j}=\left[l_{s j}^{t}, l_{o j}^{m}, F_{\max j}, S c_{j}\right]
$$

Where $j$ denotes the index of the muscle involved in the knee joint flexion or extension.

\section{B. Identification results}

The identification procedure is done with a young healthy subject with the following features (Heigth $=1.85 \mathrm{~m}$, Weight $=92 \mathrm{Kg}$, Sex=Male, Age=29 years old).

The knee joint angle $\theta$ is measured through an incremental encoder while the exoskeleton generated torque $\tau_{e}$ is computed using the actuator current sensor. Both measures $\theta$ and $\tau_{e}$ are filtered using a zero-lag fourth-order low-pass Butterworth filter with a cutoff frequency of $3 \mathrm{~Hz}$. Joint angle velocity $\dot{\theta}$ and joint angle acceleration $\ddot{\theta}$ are derived numerically from the measured knee joint position. The torque developed by 
TABLE I

IDENTIFIED LOWER LIMB PARAMETERS

\begin{tabular}{cccc}
\hline$J\left(k g . m^{2}\right)$ & $B\left(\frac{N m \cdot s}{\operatorname{rad}}\right)$ & $K\left(\frac{N m}{r a d}\right)$ & $\tau_{g}(N m)$ \\
\hline 0.7 & 2 & 4 & 18 \\
\hline
\end{tabular}

TABLE II

Lower Limb Musculos Keletal PARAmeters (-:IDENTIFIEd, *: 1]

\begin{tabular}{cccccl}
\hline Muscle & & $l_{m}^{o}(\mathrm{~cm})$ & $l_{s}^{t}(\mathrm{~cm})$ & $F_{\max }(N)$ & $S c$ \\
\hline \multirow{2}{*}{ RF } & - & 10 & 45.2 & 1314 & 1.33 \\
& $*$ & 8.4 & 34.6 & 780 & 1 \\
\hline VL & - & 8.4 & 17.2 & 2050 & 1.02 \\
& $*$ & 8.2 & 15.7 & 1870 & 1 \\
\hline VM & - & 8.24 & 12.3 & 1381 & 1 \\
& $*$ & 8.9 & 12.6 & 1295 & 1.15 \\
\hline VI & - & 8.2 & 13.3 & 1318 & 1.14 \\
& $*$ & 8.7 & 13.6 & 1235 & 1 \\
\hline SM & - & 7.2 & 37.9 & 1090 & 1.1 \\
& $*$ & 8 & 35.9 & 1030 & 1 \\
\hline ST & - & 18.1 & 24.3 & 324 & 1.08 \\
& $*$ & 20.1 & 26.2 & 330 & 1 \\
\hline BL & - & 10.7 & 30.7 & 694 & 1.07 \\
& $*$ & 10.9 & 34.1 & 720 & 1 \\
\hline BS & - & 17.1 & 9.9 & 400 & 1.04 \\
& $*$ & 17.3 & 10 & 413 & 1
\end{tabular}

the exoskeleton's actuator is computed using measurements of a current sensor.

Step and chirp knee joint desired trajectories are used in static and dynamic conditions respectively (step I of the identification procedure). The chirp signal is used due to its frequency richness. The identification results are reported in Table I. Four EMG sensors from Delsys ${ }^{T M}$ are used to measure the muscular activities of the following muscles: rectus femoris, vastus lateralis, semi-membranous/semi-tendinous and biceps femoris long head/short head. In order to remove movement artifact, EMG acquired data of the four muscles are filtered using a fourth-order recursive Butterworth filter with a cutoff frequency equal to $30 \mathrm{~Hz}$. The resulting signal is full wave rectified, and then filtered using a Butterworth low-pass filter with a $2 \mathrm{~Hz}$ cutoff frequency. The optimization is done offline using nonlinear least squares "Levenberg Marquardt" based algorithm. Elements of the vector $\chi$ have been set initially to values taken from the literature data reported in [1] and [3] except $S_{c}$ that has been initialised to 1 . Activation muscular dynamics parameters such as $\tau_{a c t}$ and $\tau_{\text {deact }}$ are taken from [34] and set to $0.02 \mathrm{~s}$ and $0.06 \mathrm{~s}$ respectively, the muscle maximum velocity $v_{\max }$ is set to $10 l_{o}^{m}$, and the muscle damping $B^{m}$ is set to 0.1 [35].

Identification results are shown in Fig. 3 and 4 It can be shown that successful knee torque estimation is obtained, the estimated torque is close to the measured one. The identified parameters are shown in Table II; one can note that the identified parameters and those taken from literature (physical quantity) are close and the difference lies within the tolerated physiological variation [12].

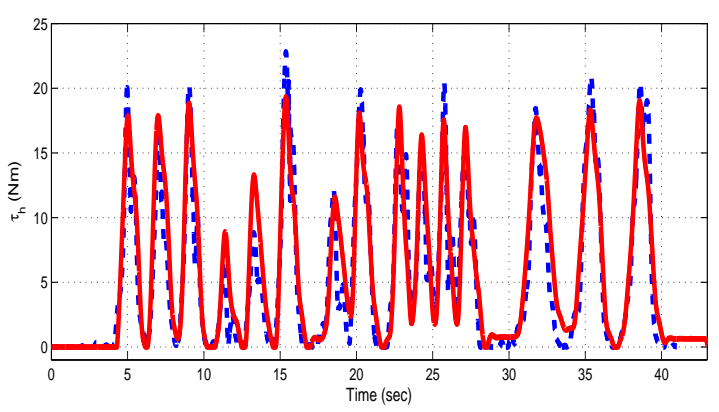

Fig. 3. Identification results of the quadriceps muscle parameters, estimated torque: (dashed line) and measured torque: (continuous line)

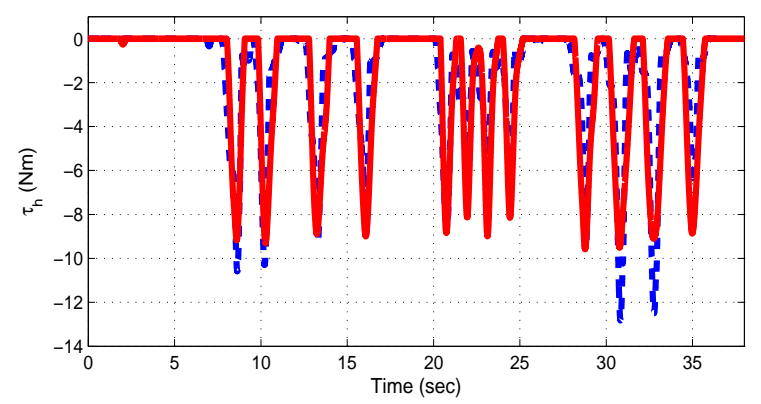

Fig. 4. Identification results of the hamstring muscle parameters, estimated torque: (dashed line) and measured torque: (continuous line)

\section{EXPERIMENTATION AND ROBUSTNESS TESTS}

This section describes the experiments conducted with a healthy subject (the same one as in the identification process) in a sitting position wearing an exoskeleton under the form of an actuated knee joint orthosis. The lower limb moves freely around the knee joint allowing flexion/extension of the shank-foot-orthosis upon the wearer's intention. The orthosis is equipped with a Maxon ${ }^{T M}$ brushless DC motor (BLDC) and an incremental encoder measuring the angle of the shank segment with respect to the thigh one. It is controlled through a PC using the dSPACE DS1103 controller board, running at $1 \mathrm{KHz}$, and the Maxon ${ }^{T M}$ motion controller EPOS 70/10. The latter drives the brushless DC motor and acts as current regulator. The velocity of the Shank-foot-orthosis is derived numerically from the measured current knee joint position.

The general control layout is illustrated in Fig. 2, the wearer's torque is estimated using the lower limb anatomical model based on the use of the modified hill-type muscle model. The raw EMG signals, captured from the quadriceps and hamstring muscles, are processed according to the procedure described in section III.

The controller parameters $\lambda_{0}$ and $K_{d}$ are tuned experimentally in passive mode to obtain the best tracking performance. In this mode, the subject is asked to not develop any muscular effort during the experiments. The best tracking performance is observed for the following controller parameter values: 

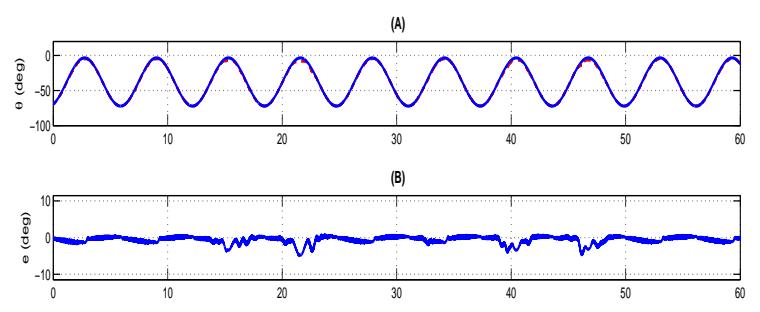

(C)

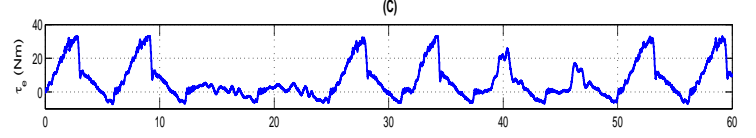

(D)
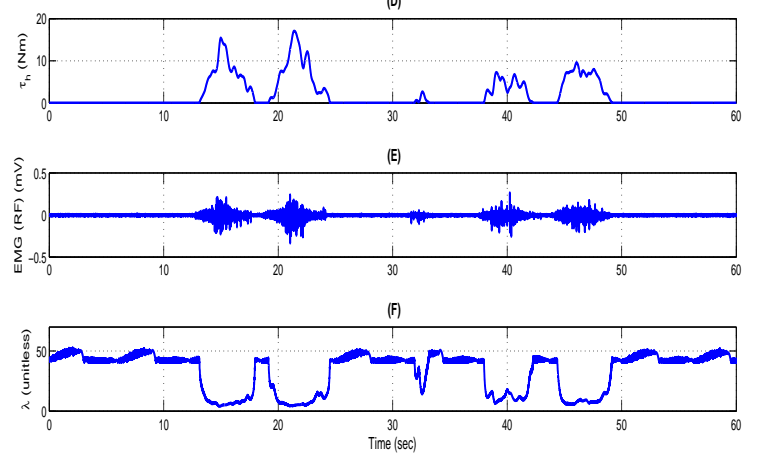

Fig. 5. Knee joint position (A), position tracking error (B), exoskeleton's torque $(\mathrm{C})$, wearer's torque $(\mathrm{D})$, rectus femoris EMG $(\mathrm{E})$ and controller's gain parameter $\lambda(\mathrm{F})$. In plot $(\mathrm{A})$ current position value is plotted in blue line while the desired one is plotted in continuous red line

$\lambda_{0}=40$ and $K_{d}=20$. In these experiments, the parameters $K_{1}$ and $K_{2}$ are set to 10 and 1 respectively.

In these experiments, only wearer's voluntary muscular contractions are considered. The experiments consist of tracking a sinusoidal desired trajectory. Three main cases are studied: in the first one, the subject is in passive mode; in the second one, the subject develops an important torque; and in the third case, the subject develops a less important torque. Fig 5 shows desired and current knee joint position (A); the position tracking error (B); the exoskeleton generated torque (C); the wearer's developed torque (D); muscular activity of the rectus femoris muscle (E) and controller's gain parameter $\lambda(\mathrm{F})$.

In the time intervals $[0 s-12 s]$ and $[25 s-32 s]$, the subject is in passive mode. An accurate tracking is observed. The tracking error is relatively small and the RMS error is equal to $0.7^{\circ}$. The assistance torque developed by the orthosis reaches the value of $32 \mathrm{Nm}$. In the interval of time [12s-24s], we observe that the subject develops a muscular activity, visible through the variation of EMG signals. The resulting torque developed by the subject reaches $17 \mathrm{Nm}$. At the same time, the assistance torque provided by the orthosis decreases and does not exceed $5 \mathrm{Nm}$. In the time interval [32s-49s], the subject develops a new muscle activity but with less intensity than in the interval
$[12 s-24 s]$. In this last case, the torque developed by the wearer does not exceed $9.5 \mathrm{Nm}$ while the orthosis provides a greater assistance torque that can reach $24 \mathrm{Nm}$. These results demonstrate clearly the high efficiency of the proposed control strategy.

\section{A. Robustness with respect to disturbances}

A wrong movement at the knee joint level may cause instability. Therefore, an important property of the proposed control law is to regain the intended position whenever an unpredictable flexion or extension occurs. This means that the control law should be robust with respect to external disturbances and to the undesirable human movements that may affect the knee joint and consequently the whole stability and safety of the wearer. In order to test the robustness of the proposed control approach, the subject is asked to apply voluntary disturbances resistive to the desired movement of the orthosis. As shown in Fig. 6 in the time interval [4.5s-7.5s], the subject applies a voluntary disturbance to resist the flexion movement of the orthosis. This is confirmed by the positive torque developed by the subject and that can reach up to $18 \mathrm{Nm}$. One can observe that the parameter $\lambda$ decreases from 50 to 13 when the tracking error increases. Once the voluntary disturbance is off, the parameter $\lambda$ regains its initial value. External involuntary disturbances that may occur during movements such as spasms can also be identified through the tracking error and can be tackled with the proposed control strategy.

In the time interval $[14 s-16.5 s]$, the wearer resists to the exoskeleton extension, this can be clearly observed by the negative torque generated by the wearer (up to $-10 \mathrm{Nm}$ ). The parameter $\lambda$ increases as the tracking error increases. The maximum value of $\lambda$ is equal to 160 and is obtained when the tracking error is equal to 60 degrees. The exoskeleton regains the desired trajectory while the value of $\lambda$ regains its initial value.

In the time interval $[20.5 s-22.5 s]$, the wearer resists to the desired movement of the exoskeleton extension with a human torque equal to $-5 \mathrm{Nm}$. Here again, the parameter $\lambda$ increases as the tracking error increases. The maximum value of $\lambda$ is equal to 100 and is obtained when the tracking error is equal to 17 degrees. The exoskeleton regains again the desired trajectory.

\section{CONCLUSion}

The present paper treated the control of a knee-joint exoskeleton driven through the wearer's intention. For this purpose, a bio-inspired musculoskeletal model is used to control the exoskeleton. Identification of the musculoskeletal model parameters, such as muscle-tendon length and developed force, is done through an unconstrained optimization problem formulation. A control law strategy based on the following rule "Assisting the subject only as much as needed to accomplish a given task", has been proposed and its asymptotic stability has been proved using Lyapunov analysis. The proposed control law is robust with respect to external 

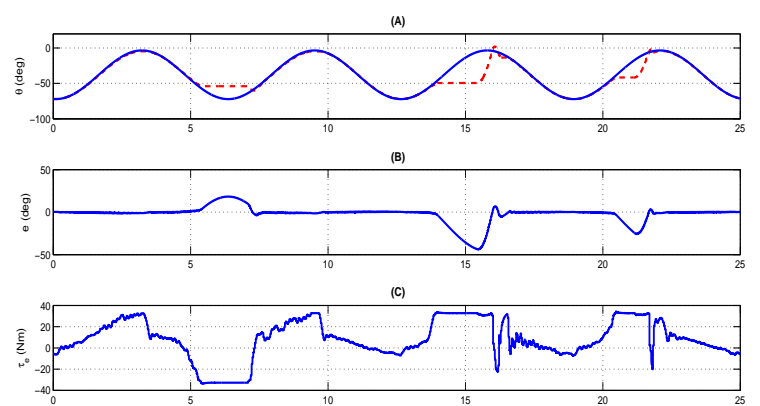

(D)

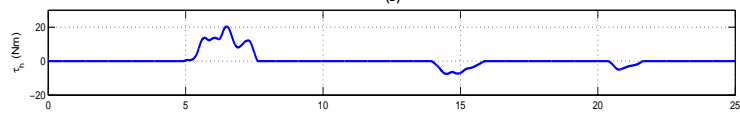

(E)

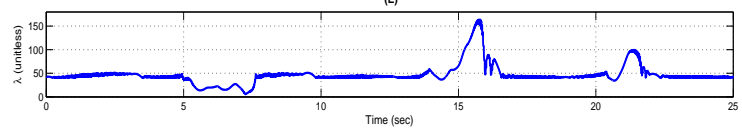

Fig. 6. Knee joint position (A), exoskeleton's torque (B), wearer's torque (C) and controller's gain parameter $\lambda(E)$. In plot (A) current position value is plotted in blue line while the desired one is plotted in continuous red line

disturbances. Experimental validations were conducted online on a healthy subject during flexion and extension of the knee joint. Satisfactory performances were observed in terms of adaptation to the desired task achievement. Robustness of the proposed control strategy with respect to errors in modeling as well as errors in parameter estimation when conducting experiments with different wearer's profiles. Future works concern the enhancement of the proposed strategy to complex tasks of daily living activities such as standing up and sitting down as functions of the wearers intention.

\section{REFERENCES}

[1] E.M. Arnold, S.R. Ward, R.L. Lieber, and S.L. Delp. A model of the lower limb for analysis of human movement. Annals of Biomedical Engineering, 38(2): 269-279, 2010.

[2] S.K. Banala, A. Kulpe, and S.K. Agrawal. A powered leg orthosis for gait rehabilitation of motor-impaired patients. In IEEE International Conference on Robotics and Automation, pages 4140-4145, 2007.

[3] S.L. Delp, J.P. Loan, M.G. Hoy, F.E. Zajac, E.L. Topp, and J.M. Rosen. An interactive graphics-based model of the lower extremity to study orthopaedic surgical procedures. IEEE Transactions on Biomedical Engineering, 37(8):757-767, 1990.

[4] C. Fleischer and G. Hommel. A human-exoskeleton interface utilizing electromyography. IEEE Transactions on Robotics, 24(4):872-882, 2008.

[5] Y. Hirata, T. Iwano, M. Tajika, and K. Kosuge. Motion control of wearable walking support system with accelerometer considering swing phase support. In IEEE
International Symposium on Robot and Human Interactive Communication, pages 322-327, 2008.

[6] M. Ishii, K. Yamamoto, and K. Hyodo. Stand-alone wearable power assist suit-development and availability. Journal of Robotics and Mechatronics, 17(5):575, 2005.

[7] T. Kawabata, H. Satoh, and Y. Sankai. Working posture control of robot suit hal for reducing structural stress. In IEEE International Conference on Robotics and Biomimetics, pages 2013-2018, 2009.

[8] H. Kazerooni, J.L. Racine, L. Huang, and R. Steger. On the control of the berkeley lower extremity exoskeleton (bleex). In IEEE International Conference on Robotics and Automation, pages 4353-4360, 2005.

[9] Z.O. Khokhar, Z.G. Xiao, C. Menon, et al. Surface emg pattern recognition for real-time control of a wrist exoskeleton. Biomedical Engineering Online, 9(1):41, 2010.

[10] K. Kiguchi, K. Iwami, M. Yasuda, K. Watanabe, and T. Fukuda. An exoskeletal robot for human shoulder joint motion assist. IEEE/ASME Transactions on Mechatronics, 8(1):125-135, 2003.

[11] K. Kong and M. Tomizuka. Control of exoskeletons inspired by fictitious gain in human model. IEEE/ASME Transactions on Mechatronics, 14(6):689-698, 2009.

[12] D.G. Lloyd, T.F. Besier, et al. An emg-driven musculoskeletal model to estimate muscle forces and knee joint moments in vivo. Journal of Biomechanics, 36(6):765776, 2003.

[13] BJ Makinson. Research and development prototype for machine augmentation of human strength and endurance. hardiman i project. Technical report, DTIC Document, 1971.

[14] Laura Marchal-Crespo and David J Reinkensmeyer. Review of control strategies for robotic movement training after neurologic injury. Journal of Neuroengineering and Rehabilitation, 6(1):20, 2009.

[15] S. Masahiro, E. Kiyoshi, and S. Yoshiyuki. Substitution of motor function of polio survivors who have permanent paralysis of limbs by using cybernic voluntary control. In IEEE International Conference on Robotics and Biomimetics, pages 504-509, 2009.

[16] Samer Mohammed, Yacine Amirat, and Hala Rifai. Lower-limb movement assistance through wearable robots: state of the art and challenges. Advanced Robotics, 26(1-2):1-22, 2012.

[17] United Nations. Department of Economic and Social Affairs. Population Division. World Population Ageing 2009. UN, UK distributor: Stationery Office, 2010.

[18] R. Ortega, A. Loria, P.J. Nicklasson, and H.J. SiraRamirez. Passivity-based control of Euler-Lagrange systems: mechanical, electrical and electromechanical applications. Springer, 1998.

[19] J.L. Pons et al. Wearable robots: biomechatronic exoskeletons, volume 70. Wiley Online Library, 2008.

[20] J.E. Pratt, B.T. Krupp, C.J. Morse, and S.H. Collins. The roboknee: an exoskeleton for enhancing strength and 
endurance during walking. In IEEE International Conference on Robotics and Automation, volume 3, pages 2430-2435, 2004.

[21] Hala Rifai, Walid Hassani, Samer Mohammed, and Yacine Amirat. Bounded control of an actuated lower limb orthosis. In Decision and Control and European Control Conference (CDC-ECC), 2011 50th IEEE Conference on, pages 873-878, 2011.

[22] Hala Rifai, Samer Mohammed, Boubaker Daachi, and Yacine Amirat. Adaptive control of a human-driven knee joint orthosis. In Robotics and Automation (ICRA), 2012 IEEE International Conference on, pages 2486-2491, 2012.

[23] J. Rosen, M.B. Fuchs, and M. Arcan. Performances of hill-type and neural network muscle modelstoward a myosignal-based exoskeleton. Computers and Biomedical Research, 32(5):415-439, 1999.

[24] J. Rosen, M. Brand, M.B. Fuchs, and M. Arcan. A myosignal-based powered exoskeleton system. IEEE Transactions on Systems, Man and Cybernetics, Part A: Systems and Humans, 31(3):210-222, 2001.

[25] M. Sartori, D.G. Lloyd, M. Reggiani, and E. Pagello. A stiff tendon neuromusculoskeletal model of the knee. In IEEE Workshop on Advanced Robotics and its Social Impacts, pages 132-138, 2009.

[26] G.S. Sawicki, K.E. Gordon, and D.P. Ferris. Powered lower limb orthoses: applications in motor adaptation and rehabilitation. In International Conference on Rehabilitation Robotics, pages 206-211, 2005.

[27] L.M. Schutte. Using Musculoskeletal Models to Explore Strategies for Improving Performance in Electrical Stimulation-induced Leg Cycle Ergometry. Stanford University, 1993. URL http://books.google.fr/books?id=UWnItgAACAAJ

[28] A. Seireg and RJ Arvikar. The prediction of muscular load sharing and joint forces in the lower extremities during walking. Journal of Biomechanics, 8(2):89-102, 1975.

[29] J.J.E. Slotine and L. Weiping. Adaptive manipulator control: A case study. IEEE Transactions on Automatic Control, 33(11):995-1003, 1988.

[30] K. Suzuki, G. Mito, H. Kawamoto, Y. Hasegawa, and Y. Sankai. Intention-based walking support for paraplegia patients with robot suit hal. Advanced Robotics, 21(12): 1441-1469, 2007.

[31] D.G. Thelen et al. Adjustment of muscle mechanics model parameters to simulate dynamic contractions in older adults. Transactions-American Society of Mechanical Engineers - Journal of Biomechanical Engineering, 125(1):70-77, 2003.

[32] A. Tsukahara, R. Kawanishi, Y. Hasegawa, and Y. Sankai. Sit-to-stand and stand-to-sit transfer support for complete paraplegic patients with robot suit hal. Advanced robotics, 24(11):1615-1638, 2010.

[33] D.A. Winter. Biomechanics and motor control of human movement. Wiley, 2009.
[34] G.T. Yamaguchi. Dynamic modeling of musculoskeletal motion: a vectorized approach for biomechanical analysis in three dimensions. Kluwer Academic Publishers Norwell, MA, 2001.

[35] F.E. Zajac et al. Muscle and tendon: properties, models, scaling, and application to biomechanics and motor control. Critical reviews in biomedical engineering, 17(4): 359, 1989. 